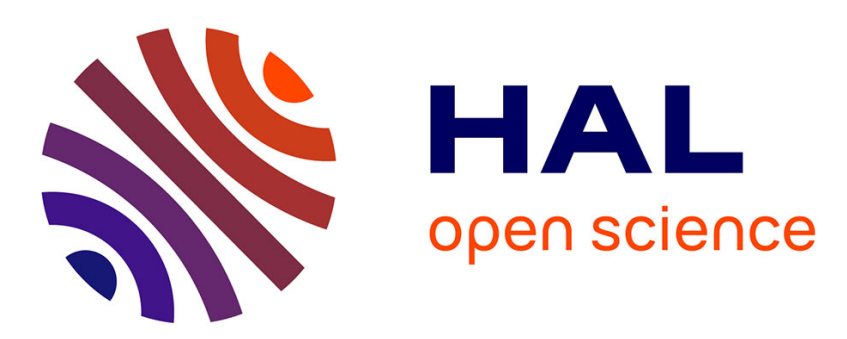

\title{
Differential flatness applied to vehicle trajectory tracking
}

Wen Chi Lu, Lili Duan, Fei-Bin Hsiao, Felix Mora-Camino

\section{To cite this version:}

Wen Chi Lu, Lili Duan, Fei-Bin Hsiao, Felix Mora-Camino. Differential flatness applied to vehicle trajectory tracking. CCC 2008, 27th Chinese Control Conference, Jul 2008, Kunming, China. pp 242-247, 10.1109/CHICC.2008.4605024 . hal-00938183

\section{HAL Id: hal-00938183 \\ https://hal-enac.archives-ouvertes.fr/hal-00938183}

Submitted on 23 Jun 2014

HAL is a multi-disciplinary open access archive for the deposit and dissemination of scientific research documents, whether they are published or not. The documents may come from teaching and research institutions in France or abroad, or from public or private research centers.
L'archive ouverte pluridisciplinaire HAL, est destinée au dépôt et à la diffusion de documents scientifiques de niveau recherche, publiés ou non, émanant des établissements d'enseignement et de recherche français ou étrangers, des laboratoires publics ou privés. 


\title{
Differential Flatness Applied to Vehicle Trajectory Tracking
}

\author{
Lu W.C. ${ }^{1}$, Duan L. ${ }^{1}$, Hsiao Fei-Bin ${ }^{1}$, Mora-Camino F. ${ }^{2}$ \\ 1.Institute of Aeronautics and Astronautics, National Cheng Kung University, Tainan, Taiwan \\ lilid925@hotmail.com; luwenchi@mail.ncku.edu.tw; fbhsiao@mail.ncku.edu.tw \\ 2. LARA, ENAC/DGAC, 7 av; Edouard Belin31055, Toulouse, France
}

felix.mora@enac.fr

\begin{abstract}
Differential flatness, a property of some dynamic systems which has been recognized only recently, has made possible the development of new tools to control complex nonlinear dynamic systems. Guidance dynamics of many different systems have been recognized as being explicitly or implicitly differentially flat as it is the case for flight guidance dynamics of conventional aircraft. In this paper, a new control structure is proposed to achieve trajectory tracking for vehicles: a neural network deals with the inversion of nominal guidance dynamics while a linear corrector cope with modelling errors and perturbations so that control directives to conventional autopilot systems can be generated in real time. The proposed approach is illustrated in the case of conventional aircraft flight dynamics.
\end{abstract}

Key Words: Nonlinear control, Differential geometry, Neural networks, Tracking, Flight control

\section{INTRODUCTION}

Differential flatness, a concept introduced by the Control School of Fontainebleau, has provided new opportunities to design advanced control and supervision schemes for non linear systems. According to this theory, given a desired trajectory for a flat output, it becomes quite easy to derive the corresponding input. Some authors have already given insight into the differential flatness of crane dynamics (Martin, 1992), vertical take-off aircraft and articulated ground vehicles (Fliess, et al., 1995) and more recently aircraft guidance dynamics (Lavigne, et al., 2003, Lu, et al. 2004).

In this communication, a control structure based on the inversion of differentially flat guidance dynamics is proposed to perform time referenced trajectory tracking. In the case of flight guidance, this approach turns effective $4 \mathrm{D}$ automatic guidance which is of utmost importance for air traffic management enhancement. To make this approach effective for real time applications, specially in the case of implicit flatness, neural networks structures appear of interest to invert the flight guidance dynamics. Issues related with the effective training of such a structure are discussed and numerical results, relative to a reference aircraft model are displayed. These results show that the proposed approach provides a new effective guidance control structure.

\section{BRIEF INTRODUCTION TO DIFFER- ENTIAL FLATNESS}

Here two definitions of differential flatness are introduced: one relative to systems for which causal relationships of interest are displayed analytically, and another one where these causal relationships are introduced through implicit functions.

Definition 1: A general nonlinear system whose dynamics are given by:

$$
\underline{\dot{X}}=F(\underline{X}, \underline{U}) \underline{X} \in \mathbf{R}^{n}, \underline{U} \in \mathbf{R}^{m}
$$

where $F$ is a smooth mapping, is said explicitly flat with respect to the output vector $\underline{Z}$, if $\underline{Z}$ is an $m^{\text {th }}$ order vector which can be expressed analytically as a function of the current state, the current input and its derivatives and also such as the state and the input vectors can be expressed analytically as a function of $\underline{Z}$ and its derivatives.

Then there exists smooth mappings $G_{X}, G_{U}$ and $G_{Z}$ such as:

$$
\left\{\begin{array}{l}
\underline{Z}=G_{Z}\left(\underline{X}, \underline{U}, \ldots, \underline{U}^{(p)}\right) \\
\underline{X}=G_{X}\left(\underline{Z}, \underline{\dot{Z}}, \ldots, \underline{Z}^{(q)}\right) \\
\underline{U}=G_{U}\left(\underline{Z}, \underline{Z}, \ldots, \underline{Z}^{(q+1)}\right)
\end{array}\right.
$$

where $p$ and $q$ are integer numbers. Vector $\underline{Z}$ is called a flat output for the nonlinear system. Although there is no systematical way to determine the flat output, the components of the flat output usually possess some physical meanings.

The explicit flatness property is of particular interest for the solution of a control problem when a physically meaningful flat output can be related with its objectives: for instance, in many situations, the control problem can be formulated as a flat output trajectory tracking problem.

However, for many systems, no complete analytical models are available to describe their full dynamics. Some of their components make use of input-output numerical devices derived both from theory and from experimental data. In these cases, the available theory provides in general the main mathematical properties of these implicit functions while experimental data is used to build accurate input output numerical devices. This happens for instance when flight dynamics modeling is 
considered either for control or simulation purposes, since in practice the aerodynamic coefficients are obtained through interpolation across large sets of look-up tables.

Definition 2: A nonlinear system given by a general implicit $\mathrm{n}^{\text {th }}$ order state representation:

$$
F(\underline{X}, \underline{\dot{X}}, \underline{U})=0, \underline{X} \in R^{n}, \underline{U} \in R^{m}
$$

where $F$ is a regular implicit mapping with respect to $\underline{\dot{X}}$, is said implicitly flat over an interior non-empty domain $\Delta \subseteq R^{n+m}$ if it is possible to find an $m^{\text {th }}$ order vector $\underline{Z}$ which meets condition equations (1) and (2) and condition:

$$
G\left(\underline{X}, \underline{U}, \underline{Z}, \underline{\dot{Z}}, \ldots, \underline{Z}^{(r)}\right)=\underline{0}
$$

where $G$ is locally invertible over $\Delta$ with respect to $\underline{X}$ and $\underline{U}$ where $r$ is an integer. Again, vector $\underline{Z}$ is said to be a flat output. The local invertibility of $G$ is guaranteed if the determinant of the Jacobian of $G$ is not zero according to the theorem of implicit functions, i.e. if:

$$
\operatorname{det}\left(\frac{\partial G}{\partial(\underline{X}, \underline{U})}\right) \neq 0
$$

In this case, given a trajectory of the flat output $\underline{Z}$, it is possible to map it numerically into the input space to derive corresponding control signals, so that one of the more interesting properties of differentially flat systems is still maintained. allows the identification of the flatness property of flight guidance dynamics, resulting in a numerical tool for new trajectory tracking applications.

\section{EXAMPLES OF FLAT GUIDANCE DY- NAMICS}

\subsection{Ground Vehicle Dynamics}

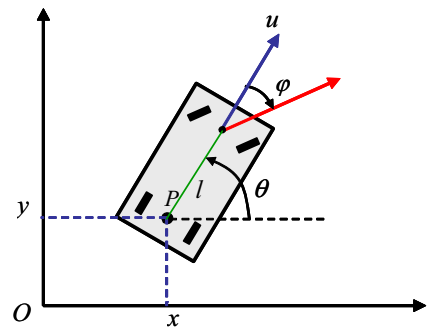

Fig.1 Ground vehicle attitude

Simplified guidance dynamics are given by :

$$
\left[\begin{array}{c}
\dot{x} \\
\dot{y} \\
\dot{\theta} \\
\dot{u}
\end{array}\right]=\left(\begin{array}{c}
u \cos (\theta) \\
u \sin (\theta) \\
0 \\
0
\end{array}\right)+\left(\begin{array}{c}
0 \\
0 \\
u / l \\
0
\end{array}\right) \operatorname{tg}(\varphi)+\left(\begin{array}{l}
0 \\
0 \\
0 \\
1
\end{array}\right) a
$$

where $(x, y, \theta, u)^{\prime}$ is a first state vector and $(a, \varphi)$ is the vector of inputs ( here $a$ is the acceleration and $\varphi$ is the orientation angle of the front wheels). Then, choosing as new state vector $(x, \dot{x}, y, \dot{y})^{\prime}$ and considering that $a$ and $\varphi$ can be expressed as:

$$
\left[\begin{array}{c}
a \\
\operatorname{tg}(\varphi)
\end{array}\right]=\left(\begin{array}{c}
\left.(\ddot{x}+\ddot{y}) \sqrt{\left(\dot{x}^{2}+\dot{y}^{2}\right)} /(\dot{x}+\dot{y})\right) \\
(\dot{x} \ddot{y}-\ddot{x} \dot{y}) /\left(\dot{y}(\dot{x}+\dot{y}) \sqrt{\left(\dot{x}^{2}+\dot{y}^{2}\right)}\right)
\end{array}\right)
$$

it is easy to show that $\mathrm{x}$ and $\mathrm{y}$ are explicit differential flat outputs for this system.

\subsection{Vertical Take-off Aircraft}

Consider the vertical dynamics of a vertical take-off aircraft (Lu, 2005) as depicted in Fig. 2:

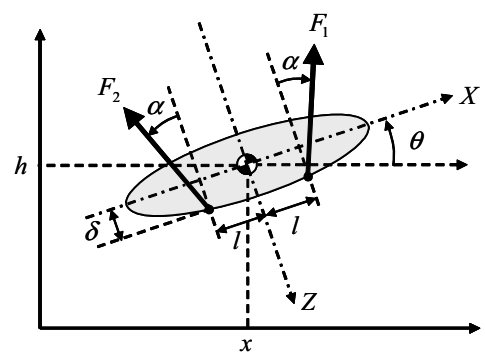

Fig.2 Vertical take-off attitude definitions

Neglecting aerodynamic effects, its motion equations are given by:

$$
\left\{\begin{array}{l}
\ddot{X}=-\left(F_{1} \sin (\theta-\alpha)+F_{2} \sin (\theta+\alpha)\right) \\
\ddot{H}=F_{1} \cos (\theta-\alpha)+F_{2} \cos (\theta+\alpha)-g \\
J \ddot{\theta}=\left(F_{1}-F_{2}\right)(l \cos \alpha+\delta \sin \alpha)
\end{array}\right.
$$

where

$$
X=x / m \quad H=h / m
$$

are the reduced coordinates of the centre of gravity of the aircraft, $m$ is the mass, $J$ is the pitch inertia moment, $l$ and $\delta$ are fixed lengths and $\alpha$ is a constant angle. It is also supposed that forces $F_{1}$ and $F_{2}$ are such as:

$$
F_{1}+F_{2}=1 / 2 \mathrm{mg} / \sin \alpha
$$

This condition implies that $\theta$ is solution of equation:

then:

$$
-\ddot{X} \sin \theta+(\ddot{H}+g) \cos \theta=1 / 2 m g / \operatorname{tg} \alpha
$$

$$
\theta=\arcsin \left(\frac{\ddot{H}+g}{\sqrt{\ddot{X}^{2}+(\ddot{H}+g)^{2}}}\right)-\arcsin \left(\frac{m g}{2 \operatorname{tg} \alpha \sqrt{\ddot{X}^{2}+(\ddot{H}+g)^{2}}}\right)
$$

so that it is easy to show that $X$ and $Y$ are explicit differential flat outputs for this system.

\subsection{Flatness of Guidance Dynamics of Conventional Aircraft}

The drag, lift and heading guidance equations for a conventional aircraft are given by ( Etkin and Reid, 1996):

$$
\dot{V}_{a}-\frac{-D+T \cos \alpha-m g(-\cos \alpha \sin \theta+\sin \alpha \cos \phi \cos \theta)}{m}=0
$$




$$
\begin{gathered}
\dot{\gamma}-\frac{L \cos \phi+T \sin \alpha-m g(\sin \alpha \sin \theta+\cos \alpha \cos \phi \cos \theta)}{m V_{a}}=0 \\
\dot{\psi}-\left(\frac{g}{V}\right) \tan \phi=0
\end{gathered}
$$

The path and heading angles are defined respectively as:

$$
\begin{gathered}
\gamma=-\sin ^{-1}\left(\dot{z} / \sqrt{\dot{x}^{2}+\dot{y}^{2}+\dot{z}^{2}}\right) \\
\psi=\tan ^{-1}(\dot{y} / \dot{x})
\end{gathered}
$$

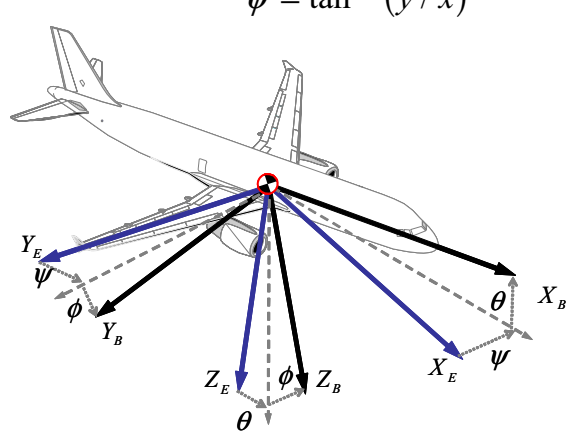

Fig.3 Aircraft angular attitude definitions

The drag force $D$ and lift force $L$ are respectively assumed to be functions of altitude $z$, airspeed $V_{a}$ and angle-of-attack $\alpha$, while the thrust $T$ can be considered to be a function of altitude $z$, airspeed $V_{a}$ and engine regime, $N_{l}$ :

$$
D=D\left(z, V_{a}, \alpha\right), \quad L=L\left(z, V_{\alpha}, \alpha\right), \quad T=T\left(z, V_{\alpha}, N_{1}\right)
$$

Considering the output $\underline{Z}=(x, y, z)^{T}$ where $\mathrm{x}, \mathrm{y}$ and $\mathrm{z}$ are the coordinates of the centre of gravity of the aircraft, and the reference inputs for the autopilot $\underline{U}=\left(\theta, \phi, N_{1}\right)$, equations (15), (16) and (17) can be rewritten as:

$$
G_{N_{1}}(\underline{Z}, \underline{\dot{Z}}, \underline{\ddot{Z}}, \underline{U})=0, G_{\theta}(\underline{Z}, \underline{\dot{Z}}, \underline{\ddot{Z}}, \underline{U})=0, G_{\phi}(\underline{Z}, \underline{\dot{Z}}, \underline{\ddot{Z}}, \underline{U})=0
$$

These implicit functions are locally invertible with respect to the input vector since for normal flight conditions the determinant of their Jacobian is not zero ( $\mathrm{Lu}$, et al. 2004) :

$$
\left|\frac{\partial G}{\partial U}\right| \neq 0
$$

then, $x, y$ and $z$ are implicit differential flat outputs for the guidance dynamics of a conventional aircraft.

Observe that in all the above examples, the coordinates of the vehicle reference point are flat outputs involved up to their second derivatives.

\section{THE PROPOSED CONTROL STRUCTURE}

As a consequence of the above flatness property, given a smooth reference trajectory (here we consider 3D trajectories) for the coordinates of the centre of gravity of a vehicle taken as flat outputs :

$$
\underline{Z}_{c}(\tau)=\left(x_{c}(\tau), y_{c}(\tau), z_{c}(\tau)\right)^{T}, \tau \in\left[t_{0}, t\right]
$$

The corresponding reference input values at the instant $t, \underline{U}_{c}(t)$, are solutions of a set of equations extracted from the guidance dynamics:

$$
\underline{G}\left(\underline{Z}_{c}(t), \underline{\dot{Z}}_{c}(t), \underline{Z}_{c}(t), \underline{U}_{c}(t)\right)=\underline{0}
$$

where $\underline{Z}_{c}(t), \underline{Z}_{c}(t)$ and $\underline{Z}_{c}(t)$ are the current reference parameters. When these equations are implicit or when they are too intricate, it is difficult to get an accurate online numerical solution. Then, it may appear of interest to build a neural network with this objective. Once correctly trained, this neural network should be an efficient computing device where the inputs are provided by the tracked trajectory, while the outputs of the neural network are reference values for the control parameters. Multilayer feed forward neural networks have been shown (Haykin, 1994) to be able to perform this task.

However a mere inversion of guidance dynamics results in an open loop control structure unable to cope with modeling and inversion errors and with external perturbations, resulting in an uncontrollable trajectory drift. Thus a corrective feedback loop must be added to guarantee guidance accuracy. This leads to the following control structure:

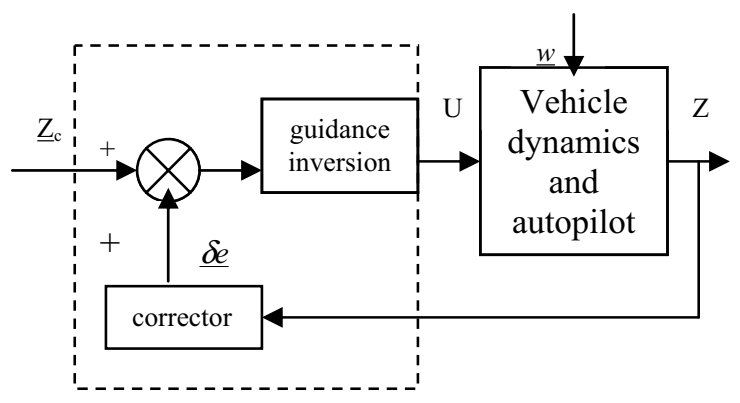

Fig.4 Proposed guidance control structure

Depending of the adopted model for the considered guidance dynamics, the guidance inversion will be performed analytically or through a neural network.

\section{THE DESIGN OF THE CORRECTIVE LOOP}

To design the corrective loop a linear model of the dynamics of the drift can be adopted. The guidance dynamics can be written in general as

$$
\begin{aligned}
\underline{\dot{X}} & =f(\underline{X}, \underline{Z}, \underline{U}) \\
\underline{Z} & =g(\underline{X})+\underline{w}
\end{aligned}
$$

with $\underline{Z}=(x, y, z)^{\prime} \quad \underline{w}=\left(w_{x}, w_{y}, w_{z}\right)^{\prime}$ where $\underline{w}$ is the perturbation vector.

Then, starting from nominal values, a first order approximation of the drift dynamics is given by:

$$
\underline{\delta \dot{X}}=\frac{\partial f}{\partial \underline{X}} \underline{\delta X}+\frac{\partial f}{\partial \underline{U}}(\underline{X}, \underline{U}) \underline{\delta U}
$$


and

$$
\underline{\delta \dot{Z}}=\frac{\partial g}{\partial \underline{X}}(\underline{X}) \underline{\delta X}+\underline{w}
$$

Assume that around the reference conditions $(\underline{Z}(0), \underline{X}(0))$, a first order output error resulting from the guidance inversion operation $G I$, is given by:

$$
G I(\underline{e}+\underline{\delta e})=G I^{*}(\underline{e})+G I(\underline{Z}(0), \underline{X}(0)) \underline{\delta e}+\underline{\sigma}
$$

where $G I^{*}(e)$ is the unknown exact output for input $\underline{e}$, $\delta e$ is the input deviation from reference and $\sigma$ is a bias. Then the control input error is such as :

$$
\underline{\delta U}=K(\underline{Z}(0), \underline{X}(0)) \underline{\delta e}+\underline{\sigma}
$$

where in general the gain matrix $K$ is full rank.

Assuming that $\frac{\partial g}{\partial \underline{X}}$ is non singular, eliminating $\underline{\delta X}$ we get the following fully controllable output dynamics:

$$
\left[\begin{array}{c}
\underline{\delta \dot{Z}} \\
\underline{\delta \ddot{Z}}
\end{array}\right]=\left[\begin{array}{cc}
0 & I \\
0 & M
\end{array}\right]\left[\begin{array}{l}
\underline{\delta Z} \\
\underline{\delta}
\end{array}\right]+\left[\begin{array}{c}
0 \\
N
\end{array}\right] \underline{\delta e}+\left[\begin{array}{c}
0 \\
E
\end{array}\right]
$$

with:

$$
\left.\begin{array}{c}
\left.M=\frac{\partial g}{\partial \underline{X}} \frac{\partial f}{\partial \underline{X}}\left(\frac{\partial g}{\partial \underline{X}}\right)^{-1} \quad N=\frac{\partial g}{\partial \underline{X}} \frac{\partial f}{\partial \underline{U}}\right\} G \\
\underline{E}=\frac{\partial g}{\partial \underline{X}}\left(-\frac{\partial f}{\partial \underline{X}}\left(\frac{\partial g}{\partial \underline{X}}\right)^{-1} \underline{w}+\frac{\partial f}{\partial \underline{U}} \underline{\sigma}\right)
\end{array}\right\}
$$

Now, assuming that $\underline{w}$ and $\underline{\sigma}$ are slow varying parameters, we get the fully observable dynamics from $\underline{\delta Z}$ :

$$
\left[\begin{array}{l}
\frac{\delta \dot{Z}}{\delta \ddot{Z}} \\
\dot{\dot{E}}
\end{array}\right]=\left[\begin{array}{ccc}
0 & I & 0 \\
0 & M & I \\
0 & 0 & 0
\end{array}\right]\left[\begin{array}{l}
\frac{\delta Z}{\delta \dot{Z}} \\
\underline{E}
\end{array}\right]+\left[\begin{array}{c}
0 \\
N \\
0
\end{array}\right] \underline{\delta e}
$$

and an on line estimation of $\underline{E}(t)$, written $\underline{\hat{E}}(t)$ is given by :

$$
\underline{\hat{E}}=\int_{0}^{t} L_{3}(\underline{\delta Z}(\tau)-\delta \hat{Z}(\tau)) \mathrm{d} \tau
$$

with

$$
\left[\begin{array}{c}
\dot{\delta \dot{\hat{Z}}} \\
\underline{\dot{\hat{Z}}} \\
\underline{\hat{\hat{E}}}
\end{array}\right]=\left[\begin{array}{ccc}
0 & I & 0 \\
0 & M & I \\
0 & 0 & 0
\end{array}\right]\left[\begin{array}{l}
\frac{\delta \hat{Z}}{\hat{\hat{Z}}} \\
\underline{\hat{E}}
\end{array}\right]+\left[\begin{array}{c}
0 \\
N \\
0
\end{array}\right] \underline{\delta e}+\left[\begin{array}{c}
L_{1} \\
L_{2} \\
L_{3}
\end{array}\right](\underline{\delta Z}-\underline{\delta \hat{Z}})
$$

Then a PID type closed loop control term such as :

$$
\left.\underline{\delta e}(t)=-F_{\delta Z} \underline{\delta Z}(t)-F_{\delta \dot{Z}} \underline{\delta \dot{Z}}(t)-F_{E} \int_{0}^{t} \underline{\delta Z}(\tau)-\underline{\delta \hat{Z}}(\tau)\right) \mathrm{d} \tau
$$

can be adopted. The convergence condition :

$$
\lim _{t \rightarrow+\infty} \underline{\delta Z}(t)=\underline{0}
$$

is achieved when $F_{\delta Z}$ and $F_{\delta \dot{Z}}$ are chosen such as:

$$
\left[\begin{array}{cc}
0 & I \\
-N F_{\delta z} & M-N F_{\delta \dot{\alpha z}}
\end{array}\right] \text { is stable }
$$

and

$$
N F_{E}=I
$$

So we get a control law such as:

$$
\underline{U}(t)=G I\left(\underline{Z}_{c}(t)+\underline{\delta e}(t)\right)
$$

Observe here that up to 27 scalar gains can be used in the corrective term providing wide opportunities to insure robustness with respect to the tracking objective.

\section{APPLICATION TO AIRCRAFT GUID- ANCE}

A simulation study of the application of the proposed control scheme to a light aircraft, the Navion, (Schmidt, 1998 ) with a piston-propeller engine and a basic controller for attitude holding, has been performed. Here guidance inversion is performed using a neural network and a simulation model of the Navion has been used for the generation of training data and for validation purposes.

\subsection{Neural Flight Guidance Dynamics Inversion}

Here, the conventional Error-Back-Propagation neural network with only one hidden layer has been selected to perform the inversion of flight guidance dynamics, although many other neural network structures (Quiroga , 2005) have been investigated. Fig. 5 displays some of the trajectories which have been considered to generate training data.

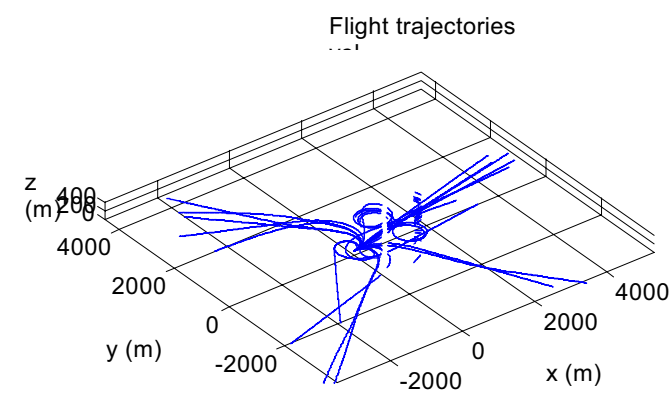

Fig.5 Neural network training trajectories

The structure of the retained neural network comprises seven inputs nodes, about 30 neurons in the hidden layer with a hyperbolic tangent activation function, and three output nodes with linear transfer functions. The inputs are altitude, three components of inertial velocity and three components of inertial acceleration. The outputs are the pitch angle $\theta$, the roll angle $\phi$ and the engine regime $N_{l}$.

Fig. 6 displays examples of training performances for different sizes of the hidden layer of the neural network. The training of this neural network, once the training data is available, appears relatively easy and provides accurate results. 


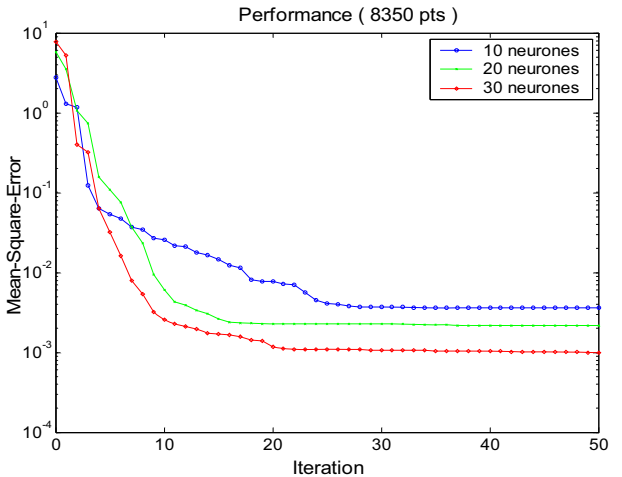

Fig.6 Training performance with different number of neurons in the hidden layer

\subsection{D Aircraft Trajectory Tracking Performances}

The next figures display simulation results for aircraft 4D trajectory tracking in two cases: in the first case no corrective term is added to the neural control scheme, while in the second case a corrective term has been introduced.

In the first case, a growing drift appears between the desired trajectory and the effective one, while in the second case, good position-time accuracy is achieved.

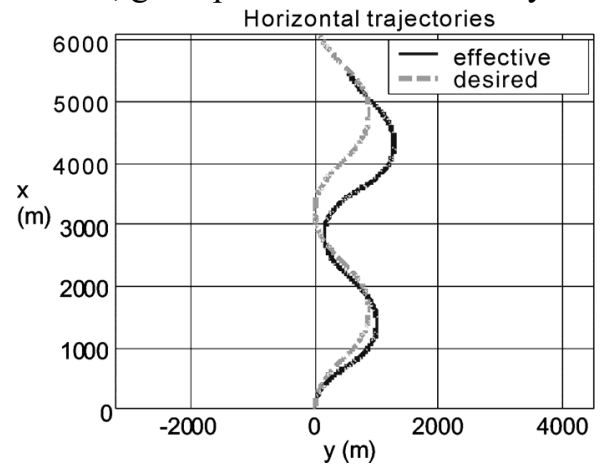

Fig.7(a) Horizontal tracks without correction

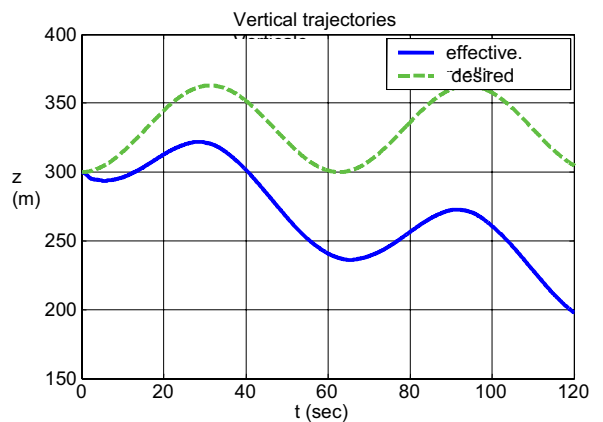

Fig.7(b) Vertical trajectories without correction

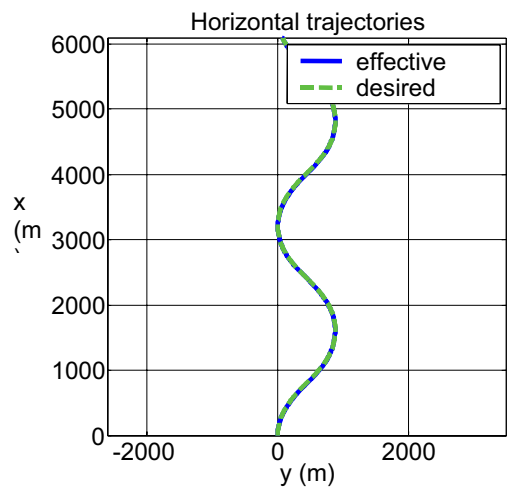

Fig.8(a) Horizontal trajectories with correction

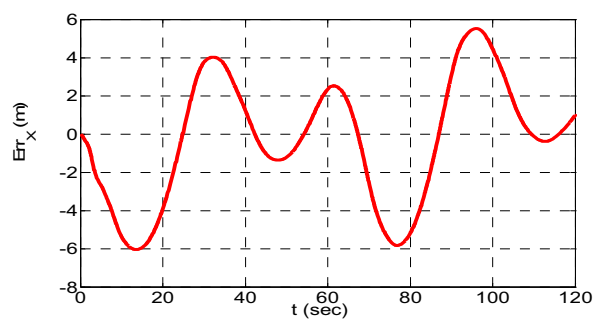

Fig.8(b) Longitudinal tracking error with correction

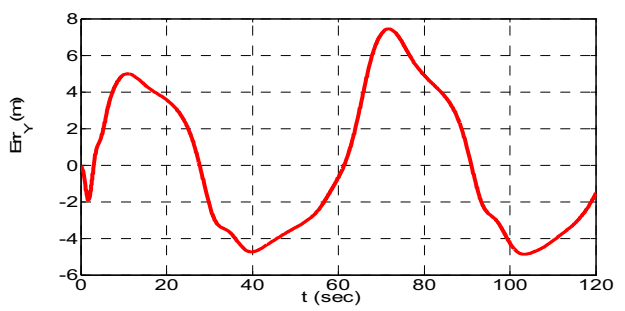

Fig.8(c) Lateral tracking error with correction

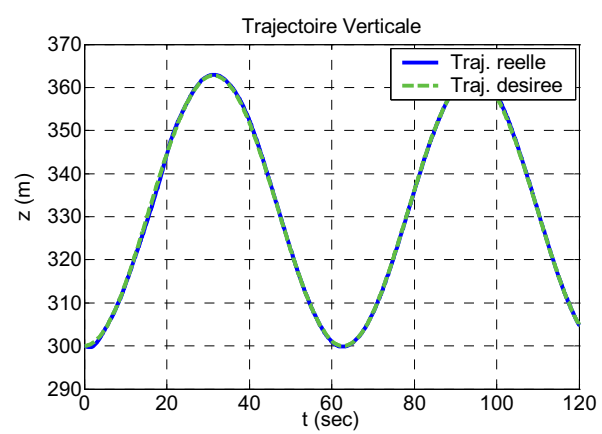

Fig.9(a) Vertical trajectories with correction

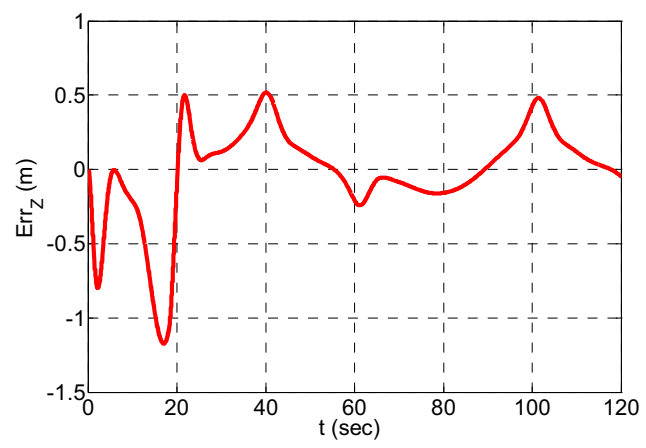

Fig.9(b) Vertical tracking error with correction

\section{CONCLUSION}

In this paper differential flatness theory has provided a sound basis for the design of effective vehicle guidance systems. The resulting control structure is independent of the tracked trajectories and thus builts up a generic guidance system. When guidance inversion is performed using a neural network, its training with a given vehicle insures that the generic guidance system is compatible with its operations domain and dynamic performances. In this paper the proposed approach has been mainly illustrated in the case of a conventional aircraft but many other potential applications are of interest. Among the many issues which remain open in this area, the design of a robust corrective term with respect to disturbances and modeling errors appears essential. 


\section{REFERENCES}

[1] ETKIN B, REID L R. Dynamics of Flight-Stability and Control. John Wiley \& Sons. New York, NY.

[2] FLIESS M, LÉVINE J, MARTIN P, ROUCHON P. Flatness and defect of non-linear systems: theory and examples. International Journal of Control, 1995,61(6): 1327-1361.

[3] HAYKIN S. Neural Networks, a Comprehensive Foundation. Macmillan Publishing Company, New Jersey.

[4] LAVIGNE L, CAZAURANG F, BERGEON B. Modelling of Longitudinal Disturbed Aircraft Model by Flatness Approach. AIAA Guidance, Navigation, and Control Conference . Austin, Texas.
[5] LU W C, MORA-CAMINO F, ACHAIBOU K. Flight Mechanics and Differential Flatness. Dynamics and Control Conference. Ilha Solteira, Brasil, 830-839.

[6] LU W C. Contribution au Suivi Automatique de Trajectoires par un Avion: Commande Plate et Réseaux de Neurones. Université Toulouse 2. PhD Thesis, Toulouse.

[7] MARTIN P. Contribution à l'étude des syst è mes differentiellement plats. Ecole des Mines de Paris. Ph.D. Dissertation, Paris.

[8] Quiroga Rodríguez, C E. Représentation Neuronale de la Dynamique de Vol d'un Aéronef. MSC/DEA report, LAAS du CNRS, Toulouse.

[9] SCHMIDT L V. Introduction to Aircraft Flight Dynamics. AIAA Education Series, Pratt Editor. 